\title{
Mantle convection interacting with magma ocean
}

\author{
R. Agrusta ${ }^{1}$, A. Morison ${ }^{1}$, S. Labrosse ${ }^{1}$, R. Deguen ${ }^{1}$, T. Alboussière ${ }^{1}$, P. J. Tackley ${ }^{2}$, F. Dubuffet ${ }^{1}$ \\ ${ }^{1}$ Université de Lyon, ENSL, UCBL, CNRS, LGL-TPE, 46 allée d'Italie, F-69364 Lyon, France \\ 2 Institute of Geophysics, Department of Earth Sciences, ETH Zürich, Sonneggstrasse 5, 8092 Zürich, Switzerland
}

Received 2019; in original form 2019

\begin{abstract}
SUMMARY
The presence of a magma ocean may have characterized the beginning of terrestrial planets and, depending on how the solidification has proceeded, the solid mantle may have been in contact with a magma ocean at its upper boundary, its lower boundary, or both, for some period of time. At the interface where the solid is in contact with the liquid the matter can flow through by changing phase, and this affects convection in the solid during magma ocean crystallization. Linear and weakly non linear analysis have shown that Rayleigh-Bénard flow subjects to two liquid-solid phase change boundary conditions is characterized by a non-deforming translation or weakly deforming long wavelength mode at relatively low Rayleigh number. Both modes are expected to transfer heat very efficiently, at least in the range of applicability of weakly nonlinear results for the deforming mode. When only one boundary is a phase change, the critical Rayleigh number is also reduced, by a factor of about 4 , and the heat transfer is also greatly increased. In this study we use direct numerical simulations in two-dimensional Cartesian geometry to explore how the solid convection may be affected by these boundary conditions for values of the Rayleigh number extending beyond the range of validity of the weakly non-linear results, up to $10^{3}$ times the critical value. Our results suggest that solid state convection during magma ocean crystallization may have been characterized by a very efficient mass and heat transfer, with Nusselt number and velocity at the least twice the value previously thought.
\end{abstract}

Key words: Mantle processes; Composition and structure of the mantle; Phase transitions; Planetary interiors; Numerical modelling

\section{INTRODUCTION}

Partial or even complete melting of the silicate mantle may have occurred early in the history of rocky planets, and depending on the phase diagram involved (e.g. Thomas et al. 2012; Boukaré et al. 2015), the solid mantle may have crystallised upwards and/or downwards leading to a solid mantle bounded above and/or below by molten layers, commonly called magma oceans (Debaille et al. 2007; Labrosse et al. 2007; Elkins-Tanton 2012; Solomatov 2015, e.g.). A similar situation is currently encountered in icy satellites like Enceladus, Europa, Titan, where the shallow icy layer is in contact with liquid water ocean and where possibly a high-pressure ice layer underlies the buried ocean (Khurana et al. 1998; Pappalardo et al. 1998; Grasset et al. 2000; Tobie et al. 2003; Baland et al. 2014; Cadek et al. 2016). The existence of such a phase change at the boundary of a solid mantle is thought to strongly affect its dynamics (Deguen 2013; Labrosse et al. 2018) and this is the subject of the present paper.

Usually, convection models in solid mantles assume a nonpenetrating boundary condition at the horizontal boundaries of the solid shell, where the free-surface boundary condition is modeled as a free-slip boundary condition on an undeformed surface. This approximation is valid as long as the dynamic topography generated by convective stresses is small and is affected only slowly by surface processes (Ricard et al. 2014). This approximation has been used for mantle convection models as it operates in the current Earth and planets (Schubert et al. 2001), but also in the presence of a magma or water ocean (Maurice et al. 2017; Ballmer et al. 2017). However, at the boundary between the solid and liquid the matter may flow through by changing phase. This requires that the latent heat released in regions of freezing (inflow for the solid) is transferred efficiently to regions where it is consumed for melting (outflow). Whether this happens depends on how fast latent heat is transferred in the liquid region compared to the rate at which topography is generated by solid viscous flow (Alboussière et al. 2010; Deguen et al. 2013; Deguen 2013). Indeed, if the heat transfer in the liquid is able to erase the topography formed by viscous deformation, the stress supply by the lithostatic stress due to the topography variation will not balance the viscous stress of the convective solid, and the liquid-solid boundary can be considered as semi-permeable (Deguen et al. 2013; Deguen 2013). This process, that leads to semi-permable boundary condition, has been shown to strongly affect the dynamics of the solid and the associated heat transfer leading for example to a translation dynamics in the Earth's inner core in contact with the liquid outer core (Alboussière et al. 2010; Monnereau et al. 2010; Deguen et al. 2013; Mizzon \& Monnereau 2013; Deguen et al. 2018), whereas only recently, attention has been paid on its effect on the evolution of the 
solid mantle (Deguen 2013; Morison et al. 2019; Labrosse et al. 2018).

Morison et al. (2019) looked at the effect of semi-permeable solid-liquid phase change boundaries on the development of the first mantle overturn during magma ocean crystallisation of the silicate mantle of the Earth, Mars, and Moon. They show that solidliquid phase change boundary conditions make the timescale of the first overturn decrease by several orders of magnitude compared to the case where solid-liquid phase change is not taken into account (Ballmer et al. 2017; Maurice et al. 2017; Boukaré et al. 2018). Moreover, Labrosse et al. (2018) performed both linear and weakly non linear analysis to show that RayleighBénard flow in a two-dimensional (2D) Cartesian geometry, subjects to one semi-permeable boundary, representing the simplest scenario during solid mantle formation, presents an heat transfer efficiency much higher than the classical values obtained with nonpenetrating boundary conditions. Moreover, their study shows that the flow is characterized by a non-deforming translation mode or weakly deforming long-wavelength mode if the flow is allowed at both boundaries of the solid mantle. Both translation and weakly deforming modes are mechanisms able to transfer heat very efficiently, and may have characterized mantle dynamics during the primordial epochs of Earth or of larger size terrestrial planets.

In this study, using 2D Cartesian numerical simulations, we explore how solid-state Rayleigh-Bénard convection may be affected by the presence of one or two solid-liquid phase change(s) at horizontal boundary(ies). We will compare and discuss the applicability of weakly non-linear results of Labrosse et al. (2018) to finite amplitude situations at high Rayleigh number, and we will discuss likely consequences of these boundaries conditions on the primordial evolution of the Earth or other terrestrial planets.

\section{METHOD}

\subsection{Governing equations}

Solid-state mantle convection is described by the system of conservation equations for mass, momentum and energy for an incompressible fluid with infinite Prandtl number and in the Boussinesq approximation. These equations, rendered dimensionless using the thickness $H$ of the solid mantle for length and the diffusion time $H^{2} / \kappa$, with $\kappa$ the thermal diffusivity for time, are:

$$
\begin{gathered}
\boldsymbol{\nabla} \cdot \mathbf{u}=0 \\
\nabla^{2} \mathbf{u}-\nabla p+\operatorname{RaT} \mathbf{z}=0 \\
\frac{\partial T}{\partial t}+\mathbf{u} \cdot \nabla T=\nabla^{2} T
\end{gathered}
$$

with $\mathbf{u}=(v, w)$ the velocity, $p$ the pressure, $T$ the temperature, $t$ the time, and $R a$ the Rayleigh number:

$$
R a=\frac{\alpha g \rho_{s} \Delta T H^{3}}{\kappa \eta},
$$

with $\alpha$ the thermal expansion coefficient, $g$ the acceleration of gravity, $\rho_{s}$ the density of the solid, $\Delta T$ the temperature difference between the lower and upper boundaries and $\eta$ the viscosity.

This set of equations neglects many of the complexities of mantle convection: in this first study, we assume a Newtonnian rheology with a constant viscosity, we do not consider volumetric heat generation, all physical parameters are assumed uniform and constant and no compositional effect is included. Although we recognise the importance of these complexities to understand mantle dynamics, in particular for recent periods (Schubert et al. 2001), we consider the simplest possible convective system to isolate the effects of the phase change boundary condition.

\subsection{Treatment of the solid-liquid phase changes}

At the boundary between a solid mantle and a liquid of the same composition, a flow through the phase change can take place. Whether the flow through the phase change takes place or not depends on the latent heat transferred through the liquid region during topography variations due to solid viscous flow (Deguen et al. 2013). Stresses in the solid lead to the formation of topography of the solid-liquid interface and convective heat transfer in the liquid tends to homogenize temperature and suppress that topography. On the one hand, if the topography is able to build because the heat transfer in the liquid region is slow, the radial velocity at interface is limited by the weight of the topography (classical dynamic topography balance) and the flow across the boundary is effectively inhibited. On the other hand, when heat transfer in the liquid is fast, it can destroy the topography by transporting heat from places where crystallization occurs to places where melting happens, and the flow through the boundary is allowed. To include this process, the solid-liquid phase change is accounted for by considering the variation in the stress field and the associated dynamic pressure at the phase boundary. Details can be found in previous papers (Deguen 2013; Deguen et al. 2013; Labrosse et al. 2018) and the derivation of the boundary condition is only shortly recalled here.

Across the solid-liquid boundary the total stress must be continuous, and if the topography slope, the viscous stress and dynamic pressure in the liquid side are assumed small, they can be neglected, and the vertical stress equilibrium acting along the undeformed phase boundary is

$$
\left(\rho_{s}-\rho_{l}^{ \pm}\right) g h^{ \pm}+2 \eta \frac{\partial w}{\partial z}-p=0 .
$$

The first term is the differential stress between the solid and liquid hydrostatic pressures, with $\rho_{s}$ and $\rho_{l}$ the solid and liquid density respectively and $h$ the topography height, the second terms and third terms $(p)$ are the viscous stress and the dynamic pressure on the solid side. The ${ }^{+}$and ${ }^{-}$exponents refer to the upper and lower boundaries, respectively. Note that Chambat et al. (2014) argue for a discontinuity of traction across the boundary and propose to add two terms to the balance equation (5). A preliminary analysis has shown that these two terms are negligible for applications to mantle convection and they are omitted here for simplicity.

At the solid-liquid boundary, like any phase change, the reaction is accompanied by release and absorption of latent heat, during freezing and melting, respectively. Because the interface between solid and liquid cannot accumulate or lose heat, the discontinuity of heat flow at the interface must equilibrate the release or absorption of latent heat due to the reaction. This may be expressed by the Stefan condition,

$$
\rho_{s} L v_{\phi}=\rho_{s} c_{p s} u_{s}\left(\frac{\partial T}{\partial z}\right)_{s} h^{ \pm}-\left[\rho_{l} c_{p l} u_{l}\left(\frac{\partial T}{\partial z}\right)_{l}\right]^{ \pm} h^{ \pm}
$$

where the term on the left represents the heat flux due to freezing or melting, with $L$ the latent heat, and $v_{\phi}$ the freezing (negative for melting) rate. The first and second terms on the right-hand-side are the heat flow from the solid to the liquid and vice-versa, respectively. $c_{p}$ is the heat capacity and $u$ the velocity, where the subscript $s$ and $l$ refer to solid and to liquid respectively. The heat flow on the 
right-hand side is dominated by the low-viscosity liquid side, and for the sake of simplicity the first term on the right side of eq. 6 is neglected. Moreover, the freezing (or melting) rate can be approximated by the vertical velocity $(w)$ across the boundary, if the topography growth rate is negligible. Under these conditions eq. 6 can be expressed as:

$$
w=\frac{\left(\rho_{l} c_{p l} u_{l}\right)^{ \pm}}{\rho_{s} L}\left(\frac{\partial T}{\partial z}\right)_{l}^{ \pm} h^{ \pm} \equiv \frac{h^{ \pm}}{\tau_{\phi}},
$$

$\tau_{\phi}$ being the characteristic phase-change time scale for transferring latent heat from region where it is released (freezing, around topography depression) to places where it is consumed (melting, around topography highs). Assuming eq. 7 and the viscous time scale

$$
\tau_{\eta}=\frac{\eta}{\left|\rho_{s}-\rho_{l}^{ \pm}\right| g H}
$$

eq. 5 becomes, in dimensionless form:

$$
\pm \Phi^{ \pm} w+2 \frac{\partial w}{\partial z}-p=0
$$

where $\Phi=\tau_{\phi} / \tau_{\eta}$ is the phase-change number and represents the ratio between the characteristic phase-change and viscous times scale. For a large value of $\Phi$, the phase change condition (eq. 9) implies a small value of $w$. This can be interpreted considering that when $\tau_{\eta} \ll \tau_{\phi}$, the topography forms in response to stress in the solid and the solid flow is limited by the buoyancy of the topography, which makes the vertical velocity effectively drop to zero at the boundary, which leads to an effectively non-penetrating classical free-slip boundary condition. On the other hand, for the opposite situation when $\tau_{\eta} \gg \tau_{\phi}$, the topography is erased at greater rate than it is generated. The removal of the associated buoyancy leads to a non-null velocity across the interface and the boundary is permeable.

\subsection{Numerical approach and set-up}

The equation described in 2.1 and 2.2 are solved using the finitevolume StagYY code (Tackley 1998). The mass and momentum equation 1 and 2 are discretized as a unique linear system of equations inverted using a direct solver for sparse matrices (UMFpack for sequential calculations, MUMPS for parallel calculations (Amestoy et al. 2001, 2006)), whereas the energy equation 3 is solved in an explicit manner, using a total variation diminishing (TVD) method for the advection term.

The mechanical boundary conditions are periodic on the vertical sides, and free-slip on the top and bottom domain boundaries, where eq. 9 is also applied. The thermal boundaries conditions are the Dirichlet condition of fixed temperature of 0 and 1 at the top and bottom, respectively. However, resolving numerically the boundary layers on the melting front of the flow at low values of the phase change number $(\Phi)$ is difficult at large Rayleigh number. Indeed, as shown by the analytical solution for the translation mode of convection when both boundaries have a phase change, a thermal boundary layer (TBL) of thickness $1 / w$ exists in the solid side (Labrosse et al. 2018) and since the velocity can be very large, it requires a huge number of grid points to be properly resolved. Moreover, even if extreme grid refinement can be used in the boundary layers, the stability of the explicit time-stepping scheme requires a extremely small timestep which renders calculations at high Rayleigh number inaccessible. The analytical solution for the translation mode and the weakly non-linear analysis for the deforming mode (Labrosse et al. 2018) show however that regions where the TBL is very thin are those where flow is toward the boundary and therefore are not prone to instabilities. Moreover, the temperature difference in these TBLs are tiny. In that sense, these regions play little role in the global dynamics and can be modeled using the theory developed by Labrosse et al. (2018). In that case, the thin TBL needs not to be resolved numerically and the Dirichlet boundary condition is replaced by an effective Robin one that depends on the vertical velocity $w$. The Dirichlet condition that applies at the boundary is replaced by a condition that applies on the interior side of the thin boundary layer. In practice, when the flow is toward the boundary ( $w<0$ at the bottom, $w>0$ at the top), the vertical temperature gradient should be null, $\partial \theta / \partial z=0$, whereas flow going away from the boundary carries the information of the boundary temperature and the Dirichlet condition is applied, $\theta=0$, with $\theta$ the deviation of temperature with respect to the steady-state conduction profile. This condition is written as:

$$
\Gamma^{ \pm} \theta+\left(1-\Gamma^{ \pm}\right) \frac{\partial \theta}{\partial z}=0
$$

where $\Gamma^{ \pm}$is a smooth approximation of the Heaviside function depending on the vertical velocity:

$$
\Gamma^{ \pm}=\frac{1}{2}\left[1+\tanh \left(\pi \frac{\mp w+\frac{w_{0}}{2}}{\frac{w_{0}}{2}}\right)\right],
$$

with $w_{0}$ the velocity range along which $\Gamma$ varies from 0 to 1 and is defined depending on the problem. For a large velocity toward the boundary $\left(w \ll-w_{0} / 2\right.$ at the bottom, $\left.w \gg w_{0} / 2\right), \Gamma \sim 0$ and we get a Neumann boundary condition, $\partial \theta / \partial z=0$, whereas for flow away from the boundary or slow flow toward the boundary, we get the classical Dirichlet boundary condition, $\theta=0$. Using eq. 10 the heat carried by diffusion across the thermal boundary layer is ignored and heat transport is done entirely by advection across the boundary. A similar approach has been already used to study the convection pattern with fast surface erosion or important magmatism in hot planets (Ricard et al. 2014). We checked that, for cases with intermediate velocity at the boundary that can be modeled using both boundary conditions, the results do not depend on the choice of boundary condition. We are therefore confident that the thermal boundary condition (10) can be used to model the phase change at high Rayleigh number.

The initial temperature conditions are described case by case in the results section 3 . The model domain has different mesh resolution depending of the problem, and it ranges from 18 to 128 elements for unit length.

\section{RESULTS}

We performed 323 simulations in 2D Cartesian coordinate (the full simulation list is presented in the supplementary material) to systematically investigate the convection style, the thermal structure and heat transfer efficiency in the solid mantle when it is bounded by one or two solid-liquid phase change boundaries. We investigate the effect of the phase change $\left(\Phi^{ \pm}\right)$and Rayleigh numbers $(R a)$, which allows us to have an overview of possible convection pattern during magma ocean crystallisation. In this first exploration, we do not consider the effects of many ingredients that are commonly thought to play a role in mantle convection: spherical geometry, volumetric heating, compositional variations, temperatureand depth- variation of physical properties. We make this choice in order to restrict this first study to a tractable set of independent 
parameters and compare the results to the well studied situation of Rayleigh-Bénard convection.

\subsection{Convection with a magma ocean above and below}

Let us first consider the situation where both the top and bottom boundaries are the seat of a phase change between the convecting solid and the magma oceans. This situation may have happened if the solid mantle crystallized from the middle, up- and downward (Labrosse et al. 2007; Thomas et al. 2012; Boukaré et al. 2015). For simplicity, we consider only the situations with an equal value of the phase change parameter at the top and bottom boundaries, which we call simply $\Phi$ for both sides.

\subsubsection{Non-deforming translation mode}

Labrosse et al. (2018) showed that a steady-state translation mode of convection can exist when both top and bottom boundaries are phase change interfaces. In that mode, a uniform purely vertical upward or downward flow in the solid is maintained by the buoyancy associated with a nearly uniform temperature, equal to that of the boundary at which the flow enters. This analytical solution is a good test of the numerical method.

To investigate the ability to develop a translation mode, we have performed numerical simulations, in a rectangular domain with aspect ratio $A=4$, with a finite small phase-change number $\Phi=0.01$ for both top and bottom sides. The choice of these parameters is justified by the fact that, for such a low value of $\Phi$, the critical Rayleigh number for the onset of the translation mode is $R a_{c t}=24 \Phi=0.24$ and this mode is favored over a deforming mode if the aspect ratio of the domain is smaller than the critical wavelength of the deforming mode, which is approximately 115 (Labrosse et al. 2018). The reduced Rayleigh number $\varepsilon_{t}=\left(R a-R a_{c t}\right) / R a_{c t}$ investigated ranges from 0.01 to 100 (Table $\mathrm{S} 1$, supplementary material). The numerical results show that steady state vertical translation occurs in the solid. The dimensionless vertical velocity $(w)$ increases with the Rayleigh number in a way that was predicted by the analytical solution for a steady-state translation (Labrosse et al. 2018) (Figure 1a). Figure 1b shows the temperature profiles obtained by the numerical simulations compared to the temperature profile predicted by the analytical solutions. Numerical solutions nicely reproduce theoretical results, and if on the one hand this prove the possibility of the translation mode when flow is allowed through both horizontal boundaries of the solid convective domain, on the other hand this validate our numerical method. The temperature profile obtained at low values of the Rayleigh $\left(\varepsilon_{t}<1\right)$ diverges from the conductive profile by an amount proportional to the velocity (Labrosse et al. 2018). At high Rayleigh numbers (high translation velocity), the profile assumes a form with a constant temperature equal to the temperature at the inflow boundary ( 0 for downward flow and 1 for upward flow), whereas on the opposite side the temperature drops (or rises) to the boundary temperature in a thermal boundary layer of thickness $\delta \sim 1 / w$ (Labrosse et al. 2018). Contrary to classical RayleighBénard convection where the flow is driven by horizontal density contrast, in the translation mode, the uniform topography of each boundary, $h=\tau_{\phi} w$, is maintained by the buoyancy associated with difference between the nearly uniform temperature and conductive profile that decreases linearly with height. Moreover, in the translation mode at high Rayleigh number heat is mainly advected by the translation, and the difference between the top and bottom conductive heat fluxes is equal to the advective heat flux (Labrosse et al.
2018). This imply that, at high Rayleigh numbers $\left(\varepsilon_{t} \gg 1\right)$, the heat flux scales linearly with the Rayleigh number, on the contrary to classical Rayleigh-Bénard convection where the heat flux scales as $R a^{\frac{1}{3}}$.

\subsubsection{Non-translating mode}

The simulations to study the non-translating modes of convection are performed in a model domain with aspect ratio equal to the critical wavelength, $A_{c}=2 \pi / k_{c}$, with $k_{c}$ the wavenumber for which the critical Rayleigh number is minimum. $A_{c}$ increases with the decrease of the phase-change number $\Phi$ as $\sqrt{128 \pi / 9 \Phi}$ for small $\Phi$ and tends to the classical $2 \sqrt{2}$ at large $\Phi$ (Labrosse et al. 2018). For this study we have investigated 5 values of $\Phi$, ranging from $10^{-1}$ to $10^{3}$, and the aspect ratio ranges from $\sim 36$ to $\sim 2.8$, respectively. Wider and narrower aspect ratios of respectively 1.5 and 0.5 times $A_{c}$ have been used, too. We performed numerical simulations with the supercritical Rayleigh number $R a_{s c}=\frac{R a}{R a_{c N t}}$ ranging from $10^{0.25}$ to $10^{3}$ (Table $\mathrm{S} 2$, supplementary material). The critical Rayleigh number $\left(R a_{c N t}\right)$ refers to the critical Rayleigh number for the non-translating mode and must not be confused with the one for the translation mode $\left(R a_{c t}=12\left(\Phi^{+}+\Phi^{-}\right)\right)$. In this study we use the subscript " $\mathrm{Nt}$ " to indicate the non-translating mode, and " $\mathrm{t}$ " the translation one. For low values of $\Phi, R a_{c N t} \simeq$ $R a_{c t}-0.3 \Phi^{2}$, whereas for high values of $\Phi, R a_{c N t}$ increases up to reach the critical value for a classical Rayleigh-Bénard convection $\frac{27 \pi^{4}}{4}$ (Labrosse et al. 2018). The limit between low and high $\Phi$ regimes can be assumed to occur where $24 \Phi=\frac{27 \pi^{4}}{4}$, i.e. at $\Phi=27.39$.

The temperature initial condition is:

$$
T(x, z)=1-z+0.05 \sin (\pi z) \cos \left(k_{c} x\right)
$$

which represents a conductive profile with a cosine perturbation of wavenumber $k_{c}$, maximum at the center $(z=0.5)$ and zero at the horizontal boundaries.

Figure 2 gives an overview of the convective flow obtained by the numerical simulations for three values of $R a_{s c}$ and three values of $\Phi$. At $\Phi=0.1$, convection is mostly characterized by alternating vertical up- and downward flow, whereas at higher $\Phi$ the flow is similar to the situation with classical free-slip boundary conditions. In particular, at $\Phi=0.1$, the solution is similar to alternating upand downward translation, whereas at $\Phi=10$ the flow is still able to pass throughout the phase changes, but it presents a substantial horizontal component compared to the lowest phase change number case. At even higher value, $\Phi=1000$, the flow across the phase-change boundaries appears completely limited and the solution ressembles the classical one for Rayleigh-Bénard convection with free-sleep boundary conditions. This behavior agrees with the prediction of the weakly non-linear analysis (Labrosse et al. 2018).

Figure 2 shows well the effect of $\Phi$ and $R a_{s c}$ on the thermal structure of the solution. For $\Phi=10^{3}$, we observe the classical behaviour of Rayleigh-Bénard convection with the thickness of boundary layers and the associated up- and down-welling currents that decreases with $R a_{s c}$. Conversely, the region between upand down-welling currents where the temperature is approximately uniform and close to 0.5 , hereafter the isothermal cores, becomes thicker as $R a_{s c}$ increases. A markedly different behaviour is observed for low values of $\Phi$ ( $\Phi \leq 0.1$ on the figure 2 ) for which the thickness of vertical currents does not relate to the thickness 
(a)

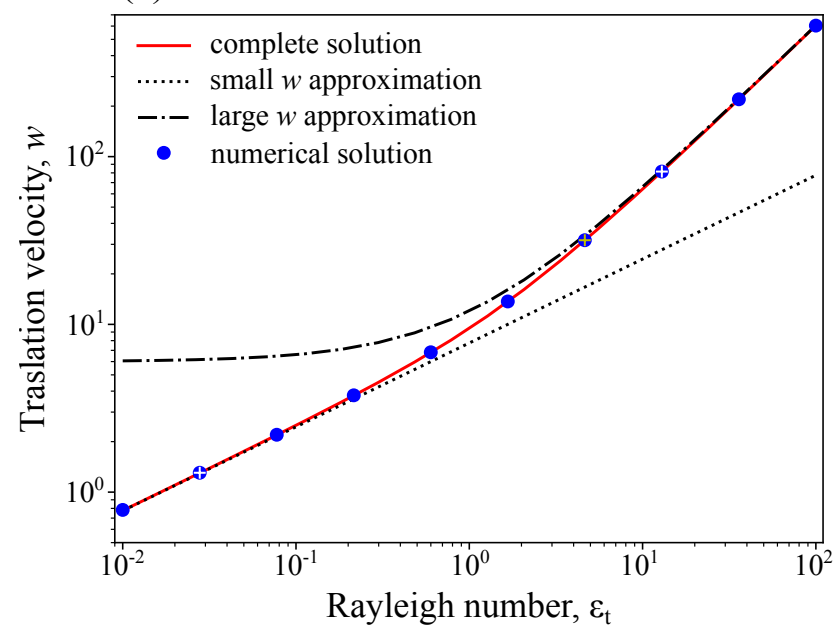

(b)

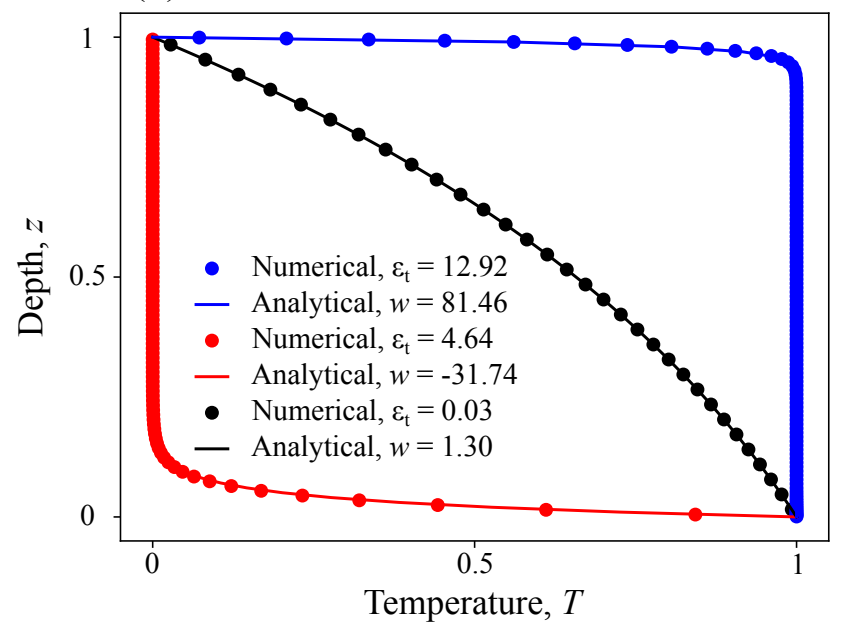

Figure 1. Non-deforming translation mode. (a) Relationship between translation velocity ( $w$ ) and reduced Rayleigh numbers ( $\varepsilon_{t}$ ) compared to the theoretical predictions (Labrosse et al. 2018). The blue symbols are the numerical simulations, and the white crosses indicate the simulations in (b). Solid, and dashed lines are the theoretical results. (b) Temperature profiles for relatively slow and fast velocities, both upward and downward compared with the theoretical profiles.
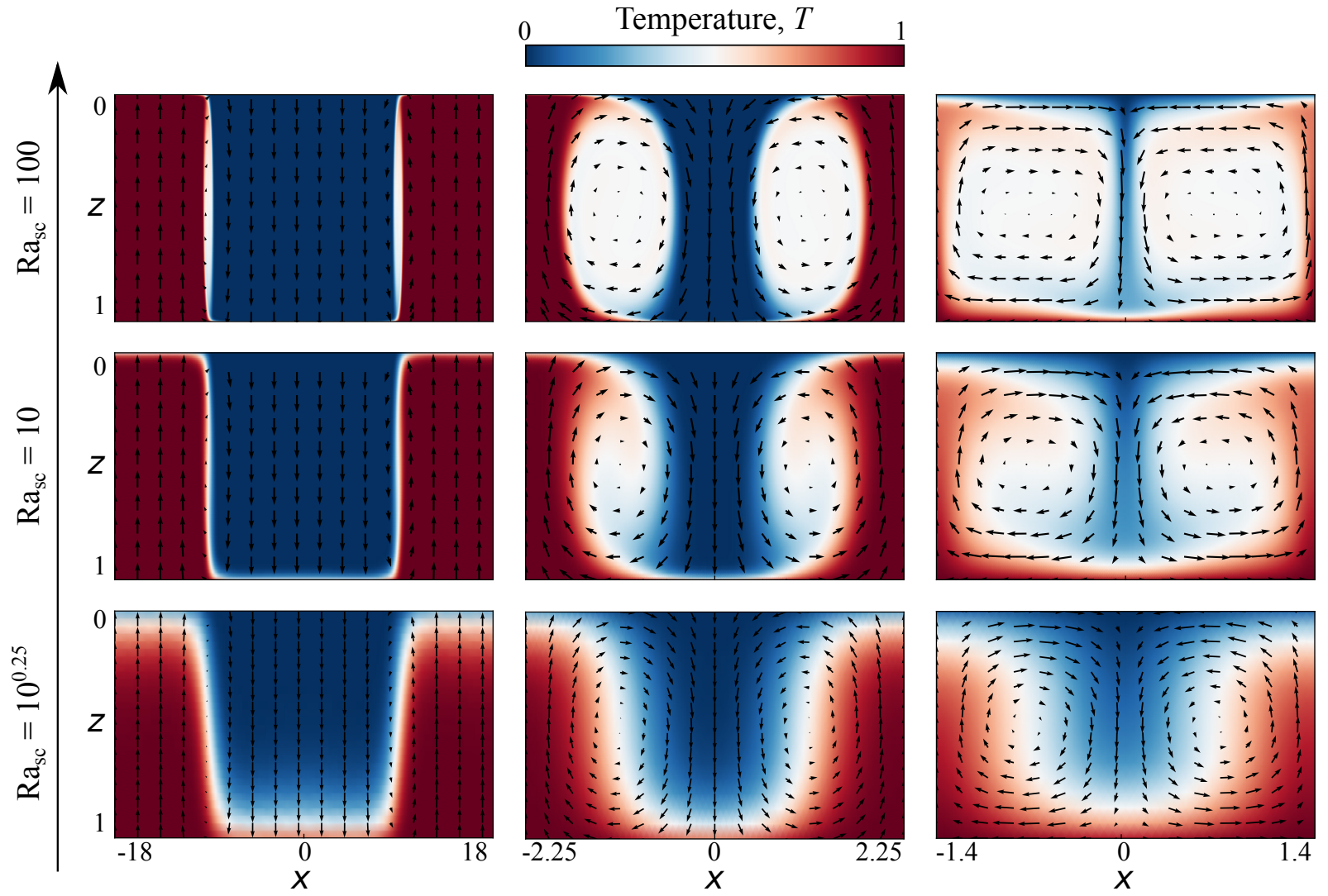

$\Phi^{ \pm}=0.1$

$\Phi^{ \pm}=10$

$\Phi^{ \pm}=1000$

Figure 2. Convection modes with two solid-liquid change boundaries. Snapshots of temperature (colour) and velocity (arrows) for different case investigated. The value of $\Phi$ increases from left to right, and the value of $R a_{s c}$ from the bottom to the top, as indicated by the axes. Note that the horizontal scale depends on the value of $\Phi$. 
of boundary layers. In addition, the boundary between the isothermal cores and the vertical currents sharpens with the increase of the Rayleigh number, and the temperature becomes more uniform in each region. To describe the thermal structure of the convective system more quantitatively, we compute the width of the isothermal core. To define the limits of the isothermal core we use the horizontal profile of the vertically averaged vertical velocity and the limits are defined where the velocity is half of the maximum value (e.g. Grigné et al. 2005). The isothermal core size normalized by the domain width is plotted against $\varepsilon_{N t}=R a_{N t}-1$ on Figure 3a. For high values of $\Phi(\Phi>100)$ the width of the isothermal core increases with the Rayleigh number. This is the behaviour typical of classical (closed boundaries) Rayleigh-Bénard convection in which plume width decreases with the Rayleigh number value, as the thickness of boundary layers from which they originate. The width of the isothermal core also increases with $R a$ at intermediate phase change number $(\Phi=10)$ but, in that case, it saturates at a value smaller than 0.5 , the maximum value that can be reached for infinitely thin plumes. On the other hand, it decreases for the smaller values of $\Phi$, leading to wider up- and down-welling currents.

For the non-translating mode solutions obtained with $\Phi$ in the low range $\Phi<27.39$, the maximum and minimum temperature profile respectively of the cold and hot current perfectly match the profile predicted by theory for the translation mode (Figure 3b), which therefore provides a good prediction of the solution for small $\Phi$. The similarity between the translation and non-translation velocity at lower $\Phi$ can be explained by the low value of the phase change number, which promotes mainly vertical flow at the expenses of the horizontal one. The numerical simulations also highlight that the non-translating mode is favored over pure translation, and prove what the linear stability analysis has suggested. Indeed the pure translation solution is unstable with respect to non translating mode, because $R a_{c N t}$ is always smaller than $R a_{c t}$ (Labrosse et al. 2018). The similarity with the translation solution disappears when $\Phi$ increases, with the transition that should occur for a value of $\Phi$ somewhere between 1 and 10. For large values of $\Phi$, the vertical flow velocity is lower than the one predicted for the translation velocity. This agrees with colder and hotter profiles respectively for the up- and down- welling current compared to the profile predicted by the translation theory (Figure 3)b. This can be explained by the fact that the difference $R a_{c t}$ and $R a_{c N t}$ increases as $\sim 0.3 \Phi^{2}$ when $\Phi$ increases, which tends to favor the non-translating mode.

To better clarify the dynamics of solid convection with two phase change boundaries, we investigate the same range of Rayleigh numbers, $\Phi$ and aspect ratio $A$, but starting from an initial temperature condition characterized by a conductive profile with a random thermal anomaly with amplitude of 0.05 (Table S3, supplementary material). Compared to the previous cases, these simulations show that the flow at low $\Phi$ can be non-symmetric, with hot and cold plumes of different widths. The horizontal wavelength of the solution can also differ from the one at the onset of convection. At higher $\Phi$ and Rayleigh number, a translation mode can also take place (Figure 4). The translation numerical solution proves what is suggested by the linear stability analysis that when the Rayleigh number increases above the critical value for the translation mode $\left(R a_{c t}\right)$ the translation mode may become the most stable solution. The dependence of the obtained solutions on the initial conditions is classical with Rayleigh-Bénard convection and we show here that, at large Rayleigh number and $\Phi$ values, a translation solution is more likely to develop than a deforming mode of convection (Ta- ble S3, supplementary material). Note that, the models presented with $\Phi=0.1$ in figure 4 despite running for a total dimensionless duration of $\Delta=8.5$ for $R a=10$ and for $\Delta t=0.05$ for $R a=100$, the system has not yet reached steady state and may still evolve toward a translation solution. In particular, the case for $\Phi=0.1$ and $R a_{s c}=100$ shows a clear asymmetry between up- and downwelling currents, the up-welling regions gaining with time. We expect it to ultimately run in an upward translation mode.

The heat transfer efficiency of the non-translating mode of convection is studied by computing the dimensionless heat flux (Nusselt number, $\mathrm{Nu}$ ) and the RMS velocity $\left(V_{r m s}\right)$, for all parameter sets investigated, but neglecting the simulations that show pure translation that have been discussed above and are already well explained by the analytical theory of Labrosse et al. (2018). In Figure 5 we show $N u$ and $V_{r m s}$ plotted against the Rayleigh number, for different values of $\Phi$. The case with classical boundary conditions and the translation velocity and the Nusselt numbers predicted by weakly non-linear analysis (Labrosse et al. 2018) are plotted for reference, too. As expected, for $\Phi=1000$, the solution roughly follows the scaling for classical Rayleigh-Bénard convection. On the other hand, for smaller values of $\Phi$, both $V_{r m s}$ and $\mathrm{Nu}$ increase more steeply with $\mathrm{Ra}$ than for non-penetrating boundary conditions. For $\Phi \leq 1$, the numerical solutions are found to closely match the prediction of the weakly non-linear analysis, for the whole range of parameters investigated, and in particular Nusselt number values in excess of $10^{3}$. This is somewhat unexpected since this first order development is only supposed to be valid very close to the onset of convection. This is another expression of the simplicity of the solution which exhibits alternative up- and downward translation regions, each very similar to the pure translation solution for which the velocity and Nusselt numbers increase linearly with $R a$ at large values of $R a$. Indeed, for each set of solution with the same value of $\Phi$ we fit the relation $N u=N u_{0} R a^{\alpha}$, and $V_{r m s}=V_{0} R a^{\beta}$, and the resulting scaling law are shown in the plot legends. In the Rayleigh number range investigated, the exponent $\alpha$ of 0.36 and $\beta$ of 0.66 for the case $\Phi=1000$ are similar to the exponents for a classical Rayleigh-Bénard convection (e.g. Jaupart \& Mareschal 2011). As $\Phi$ decreases, both exponents tend to 1 , showing the linear relationship of heat flux and velocity with the Rayleigh number, already proved for the pure translation solution (Labrosse et al. 2018). Moreover, for cases at low phase change number $(\Phi \leq 10)$ the coefficient of proportionality $N u_{0}$, and $V_{0}$, both scale as $\Phi^{-1}$, as shown for the translation mode (Labrosse et al. 2018). The heat flux and velocity obtained by the weakly non-linear analysis represent well the results from direct numerical simulations for very small value of $\Phi(\Phi \leq 1)$. On the other hand, for $10 \leq \Phi \leq 100$, the heat flux and RMS velocity from numerical solutions diverge at higher Rayleigh number values from analytical predictions, which is the usual behaviour for RayleighBénard convection.

\subsection{Convection with only one phase change boundary condition}

The situation with only one boundary having a phase change is encountered in several cases. The case with a liquid ocean below the solid layer is relevant for the surface ice-shell of some icy satellites of Jupiter and Saturn (Khurana et al. 1998; Pappalardo et al. 1998; Grasset et al. 2000; Tobie et al. 2003; Baland et al. 2014; Čadek et al. 2016) and possibly for the early Earth with a basal magma 
(a)

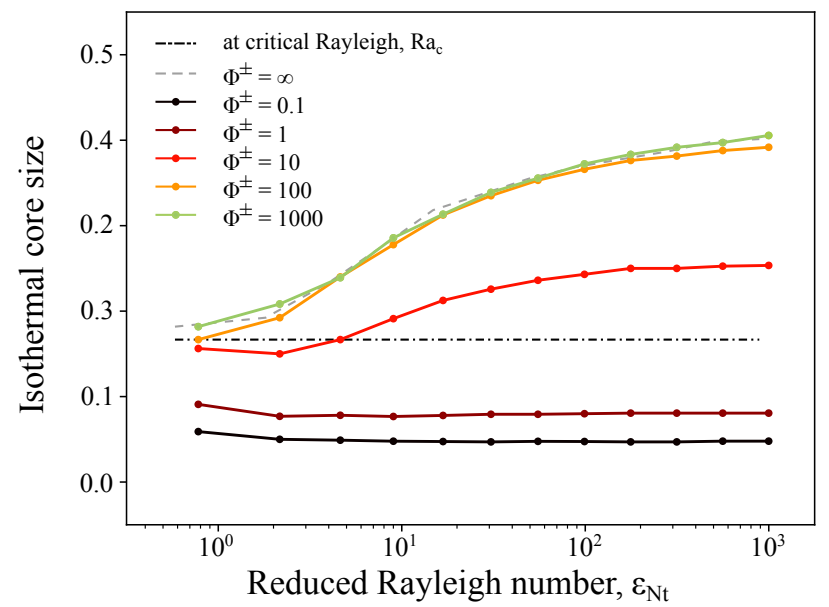

(b)

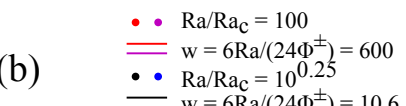

$$
\begin{aligned}
& \qquad \mathrm{Ra} / \mathrm{Ra}_{\mathrm{c}}=100 \\
& =\mathrm{w}=6 \mathrm{Ra} /\left(24 \Phi^{ \pm}\right)=134 \\
& \because \mathrm{Ra} / \mathrm{Ra}_{\mathrm{c}}=10^{0.25}
\end{aligned}
$$$$
=\mathrm{W}=6 \mathrm{Ra} /\left(24 \Phi^{ \pm}\right)=2.4
$$
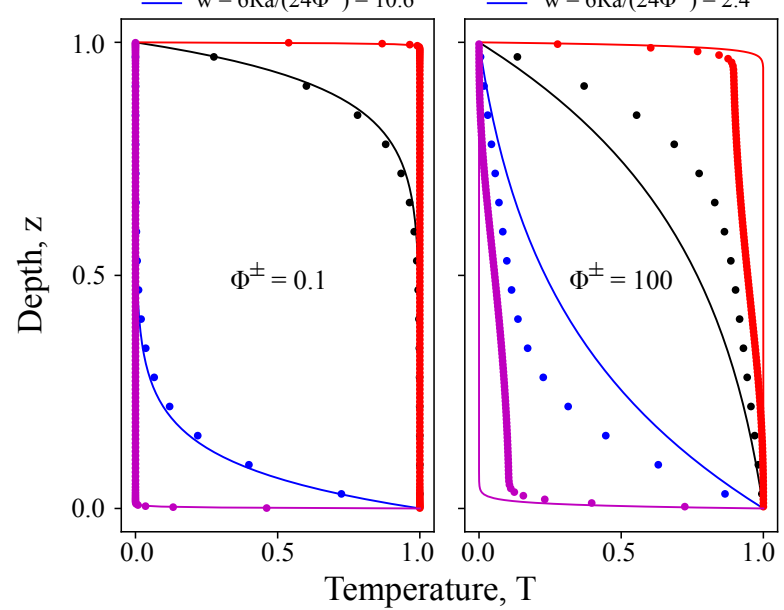

Figure 3. Thermal structure of convection with two phase change boundaries. (a) Normalized width of the isothermal core between up- and down-welling currents as function of $\varepsilon_{N t}$ (see text for details). The two dashed lines represent the width obtained by the numerical results for a classical free-slip RayleighBénard problem $\left(\Phi^{ \pm}=\infty\right)$, and at the critical Rayleigh number. (b) Temperature profiles in the up- and down-welling currents for cases at $\Phi=0.1$ and $\Phi=100$ for the $R a_{s c}$ as indicated in the legend. The dots represent the numerical solutions, whereas the solid lines are the profiles predicted by the theory for the pure translation solution for the same values of the Rayleigh number.
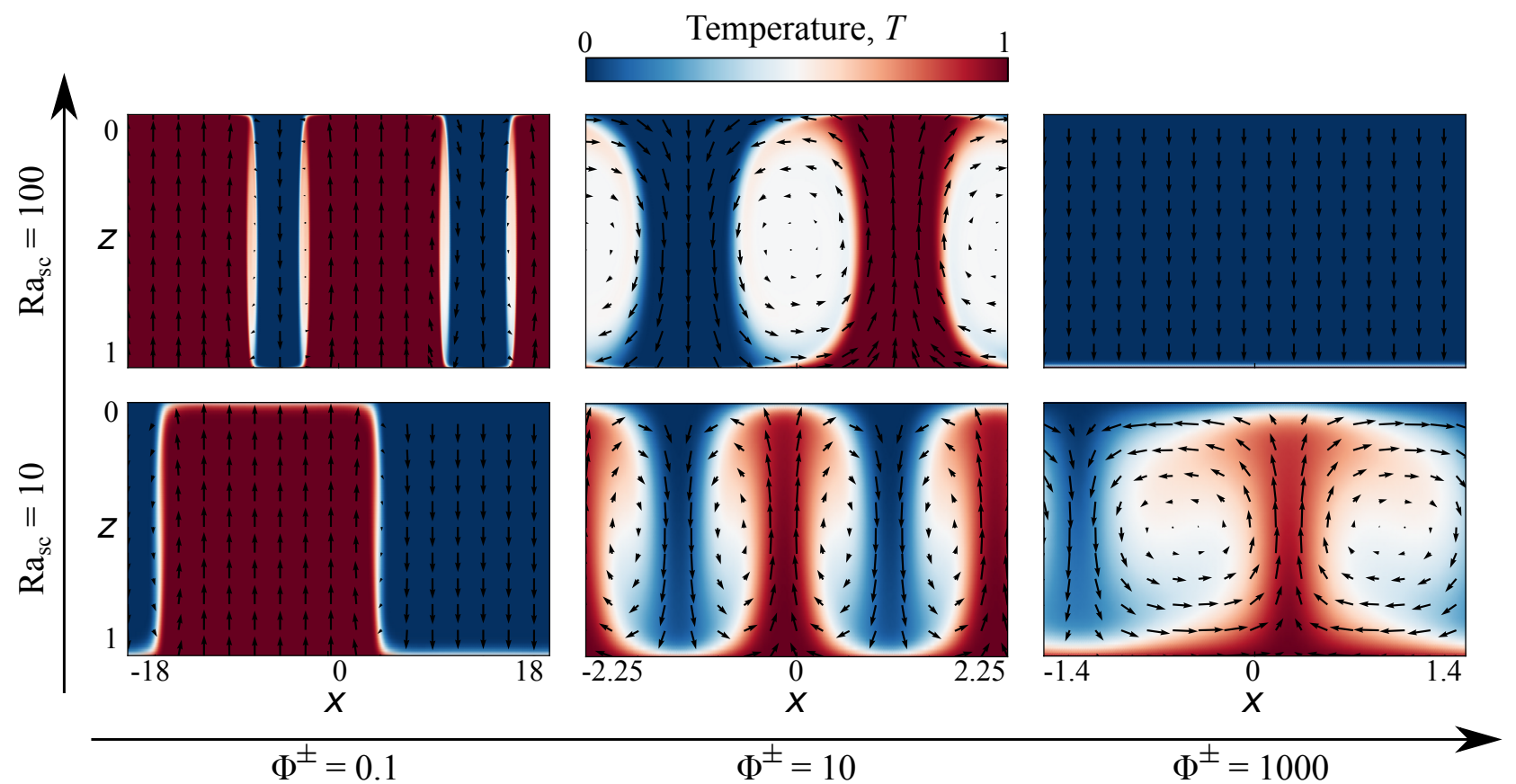

Figure 4. Convection patterns obtained with a random initial perturbation. Snapshots of temperature (colour) and velocity (arrows) for different cases investigated. The phase change parameter $\Phi$ increases from left to right, and the super-critical Rayleigh number $R a_{s c}$ from the bottom to the top, a indicated by axes. 

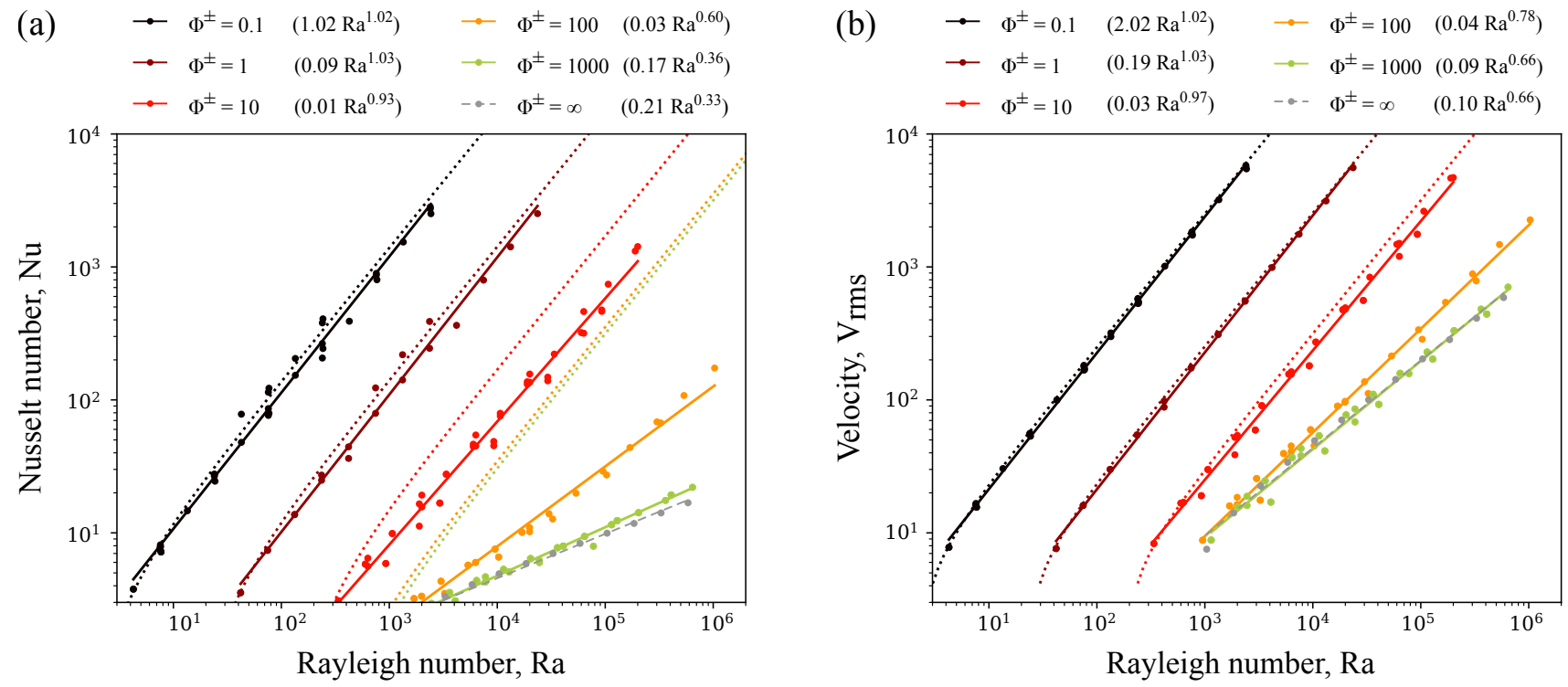

Figure 5. Heat transfer efficiency by solid-state mantle convection bounded by two magma oceans. (a) Nusselt number and (b) RMS velocity against the Rayleigh number for different values of the phase change number, as labeled. Coloured solid symbols represent results of all cases investigated. The dotted lines represent the linear (translation velocity) and weakly non-linear (heat flux) solution of Labrosse et al. (2018), whereas the gray dashed and the coloured solid lines represent the fit for each value of the phase number studied as indicated in the legend for each figures.

ocean (Labrosse et al. 2007). The case with a liquid on top of the solid may be currently relevant for high pressure ice layers below a buried ocean in the largest icy satellites (Grasset et al. 2000) and for an upwardly crystallizing magma ocean in young terrestrial planets (Solomatov 2015). In the Cartesian geometry investigated in this paper, both situations are symmetrical to one another and we only study one of them, with a magma ocean below. This is done considering a finite value for the phase change number only at the bottom boundary $\left(\Phi^{-}\right)$, while for the top one we consider $\Phi^{+}$to be infinite, in order to impose the classical non-penetrative freeslip condition. We perform the calculations using a model domain with aspect ratio equal to the critical wavelength, $A_{c}=2 \pi / k_{c}$, and imposing the same initial temperature condition as above (eq. 12 ).

Before discussing all the results, let us consider one case and discuss the symmetry between the situations with a magma ocean above and below. Figure 6 shows the final snapshots of two runs with the same parameters except for the boundary conditions, one having the $\Phi^{-}=\infty$ and $\Phi^{+}=0.1$ and the other $\Phi^{-}=0.1$ and $\Phi^{+}=\infty$. Both cases were run in a box of aspect ratio 4.978 corresponding to the wavelength of the most unstable mode at the onset of convection. After a time of about 0.05 during which convection proceeded with this initial wavelength, a transition occurred to the solution with a wavelength that is half the width of the computation domain, before resuming to a solution having the original wavelength, as displayed on the figure $6 \mathrm{a}$. As the convection proceed a periodic alternation of one and two plumes occurs. The two situations appear clearly symmetrical from one another: in the case of melting at the top, the flow is characterised by hot plumes with a cold diffuse return flow, whereas when the phase change is at the bottom, the flow is dominated by cold down-welling plumes and a diffuse hot return flow. The temperature in the return flow is equal to that of the boundary from which it originates with a boundary layer to match the opposite temperature (figure 6b). The thickness of that boundary layer controls the heat flow in that situation and its scaling is the subject of this subsection.

For our systematic study in the case of phase change condition only at the bottom, we investigated 5 values of $\Phi^{-}$, ranging from 0.1 to 1000 , with a corresponding aspect ratio between 5 and 2.8, respectively. The range of supercritical Rayleigh number $\left(R a_{s c}\right)$ is from $10^{0.025}$ to $10^{3.25}$ (Table S4, supplementary material), the lower Rayleigh number cases allowing us a detailed comparison to the predictions of the weakly non-linear stability analysis (Labrosse et al. 2018). For the computations at relatively high Rayleigh number $\left(R a_{s c} \geq 10^{2.75}\right)$ and $\Phi \leq 10$, we applied at the bottom the robin temperature boundary condition (eq. 10), assuming a threshold velocity $w_{0}$ based on the RMS velocity for a case without phase change at similar $R a_{s c}, w_{0}=0.5 V_{r m s}$. The numerical solutions for nine cases for $\Phi^{-}$of $0.1,10$ and 1000 and for $R a_{s c}$ of $10^{0.25}$, $10,10^{3}$ are shown in Figure 7. The temperature and velocity field show that at high phase-change number $\left(\Phi^{-}=1000\right)$, the solution does not differ from a classical case of non penetrating boundaries, and as $R a$ increases, the width of the isothermal core increases, which is the same behaviour we observed with phase change at both boundaries (Figure 2, and Figure 3a). At lower $\Phi^{-}$, a stationary cold plume, that becomes thinner as the Rayleigh number increases, characterizes the convective structure, and depending on the phase change number, at high Rayleigh number, a second cold plume can form, as shown in Figure 7 for $\Phi^{-}=10$ and $R a_{s c}=100$. The formation of a secondary plume occurs at $R a_{s c} \geq 10^{0.5}$ for $\Phi^{-} \leq 1$ and at $R a_{s c} \geq 10^{1.75}$ for $\Phi^{-}=1$, and the convection shows periodic alternation of one and two cold plumes. In general, the gradual increase of the Rayleigh number and/or decrease of the phase change number leads to a strong increase of the mean temperature and a consequent progressive reduction of the thickness of the top thermal boundary layer, the formation of thin and strong coldplumes, and the disappearance of the hot thermal boundary layer at the bottom. This pattern of convection is similar to that obtained for internally heated convection (e.g. Houseman 1988; Parmentier 
(a)
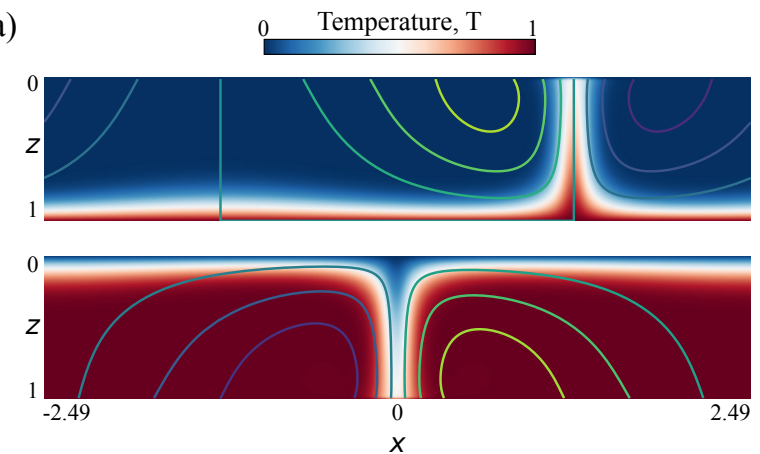

(b)

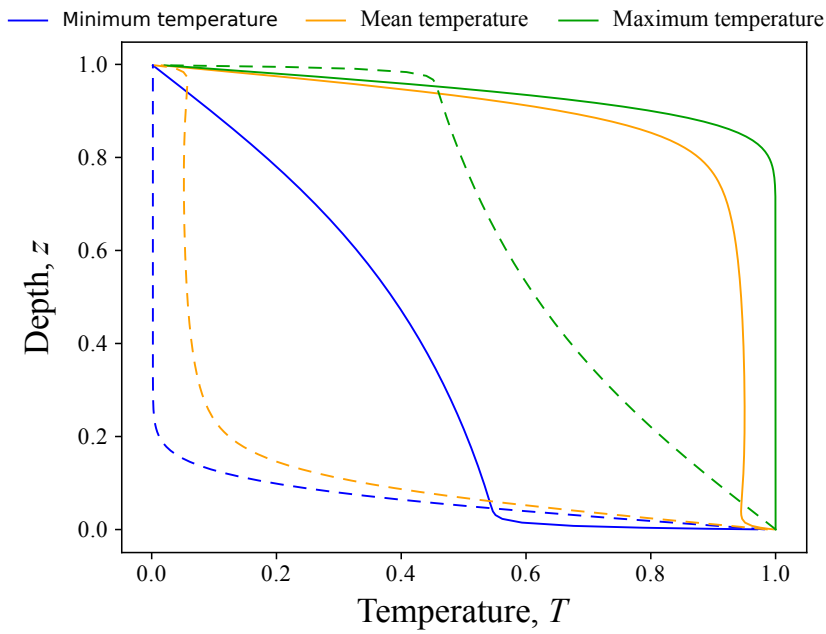

Figure 6. Solution for convection bounded by a liquid above or below. (a) Snapshots show the temperature field in colour and the streamlines at the end of the run $(t=0.18)$, (b) The vertical profiles of minimum, mean and maximum temperature. The dashed and solid lines represent the profiles for the case with a magma ocean above and below, respectively. The Rayleigh number is $R a=10^{4}$.

et al. 1994; Sotin \& Labrosse 1999), even though no volume heating is included in the present calculations.

Let us now study how the Nusselt number $(\mathrm{Nu})$ and the average temperature $(\langle T\rangle)$ vary at low Rayleigh numbers $\left(R a_{s c} \leq\right.$ $\left.10^{0.25}\right)$. Figure 8 shows $N u$ and $\langle T\rangle$ plotted against the Rayleigh number, for different values of $\Phi^{-}$, together with the prediction of the weakly non-linear analysis (Labrosse et al. 2018). The weakly non-linear analysis is found to provide good predictions only close to the critical Rayleigh number, as expected, the range of validity being somewhat larger for the average temperature than for the Nusselt number.. For large value of $\Phi^{-}$, the average temperature is close to 0.5, like for classical Rayleigh-Bénard convection, while at low $\Phi^{-}$it increases more steeply as the Rayleigh number increases. The fact that the average temperature is larger than 0.5 is again similar to the situation encountered for internally heated convection.

The Nusselt number $(\mathrm{Nu})$, the RMS velocity $\left(V_{r m s}\right)$ and the average temperature $(\langle T\rangle)$ at higher Rayleigh numbers are plotted on figure 9. The $N u$ and $V_{r m s}$ variations are bounded between the low value of the classical Rayleigh-Bénard convection with nonpenetrative conditions and the high value for low phase change number $\left(\Phi^{-}=0.1\right)$. The scaling law for the Nusselt Number, RMS velocity and temperature are, for $\Phi^{-}=0.1$ :

$$
\begin{gathered}
N u=0.37 R a^{0.33}, \\
V_{r m s}=0.2 R a^{0.66}, \\
\langle T\rangle=1.0-2.64 R a^{-0.33} .
\end{gathered}
$$

We obtained the same scaling laws for $N u$ and $\langle T\rangle$ as that obtained by Ricard et al. (2014) for mantle convection subject to fast erosion or magmatism at its surface. This indicates that different physical processes can lead to a similar physics. Moreover, as shown on figure 9, the pre-factors in the scaling laws for the Nusselt number and the RMS velocity (equations 13 and 14) are about twice their counterpart for the case with non-penetrating boundary conditions, indicating a much larger heat and mass transfer when a phase change is permitted at the boundary. As suggested by Labrosse et al. (2018), because there is not limit to vertical flow at the bottom, the Rayleigh number is equivalent to four times the Rayleigh number of the classical not-permeable case. The ratio between the pre-factors is similar to what would be expected from this simple heuristic. Results for $\Phi^{-} \geq 10$ are close to that for non-penetrative boundary conditions, at least at low values of the Rayleigh number. Increasing its value makes the heat flow at the bottom increase which makes the mean temperature increase further eventually leading to a transition to a fully open bottom boundary. This transition appears to take place at $10^{4}<R a<10^{5}$ for $\Phi^{-}=10$, and likely at higher $R a$ for $\Phi^{-} \geq 100$.

\section{CONCLUSIONS AND DISCUSSION}

In this study we have investigated the dynamics of a solid mantle bounded between two magma oceans or in contact with one at the bottom. The mantle is modeled as a two-dimensional layer of infinite Prandtl number fluid and the solid-liquid phase change at either or both boundaries is taken into account by imposing a boundary condition allowing a flow through the boundary. This boundary condition is controlled by a phase change parameter, $\Phi$, which allows the system to go from easy flow-through at low $\Phi$ values to classical non-penetration at large $\Phi$. We explored systematically the parameter space to compare with and extend the results of the weakly non-linear analysis of Labrosse et al. (2018).

For the case when the solid is bounded above and below by magma oceans, we recover the two modes of convection predicted by Labrosse et al. (2018): a steady-state up- or down-ward nondeforming translation and a deforming mode. Extending the previous results to high values of the Rayleigh number shows that the solution at small values of $\Phi$ takes the form of alternating up- and down-ward translating blocks separated by thin deformation bands. The two vertically moving blocks have a vertical velocity and a thermal structure that closely ressemble the exact analytical solution for the pure translation mode (Labrosse et al. 2018). Both convection modes are characterised by a very efficient heat transfer, in which the Nusselt number scales linearly with the Rayleigh number, whereas in the classical situation of Rayleigh-Bénard convection with non-penetrating boundary conditions, it scales as $\mathrm{Ra}^{1 / 3}$. Consequently, we find that the predictions from the weakly nonlinear analysis predict very well the behavior of the solution for the whole range of calculations performed in this study, with a Nusselt number as high as $310^{3}$.

The situation with a magma ocean above and below the solid 


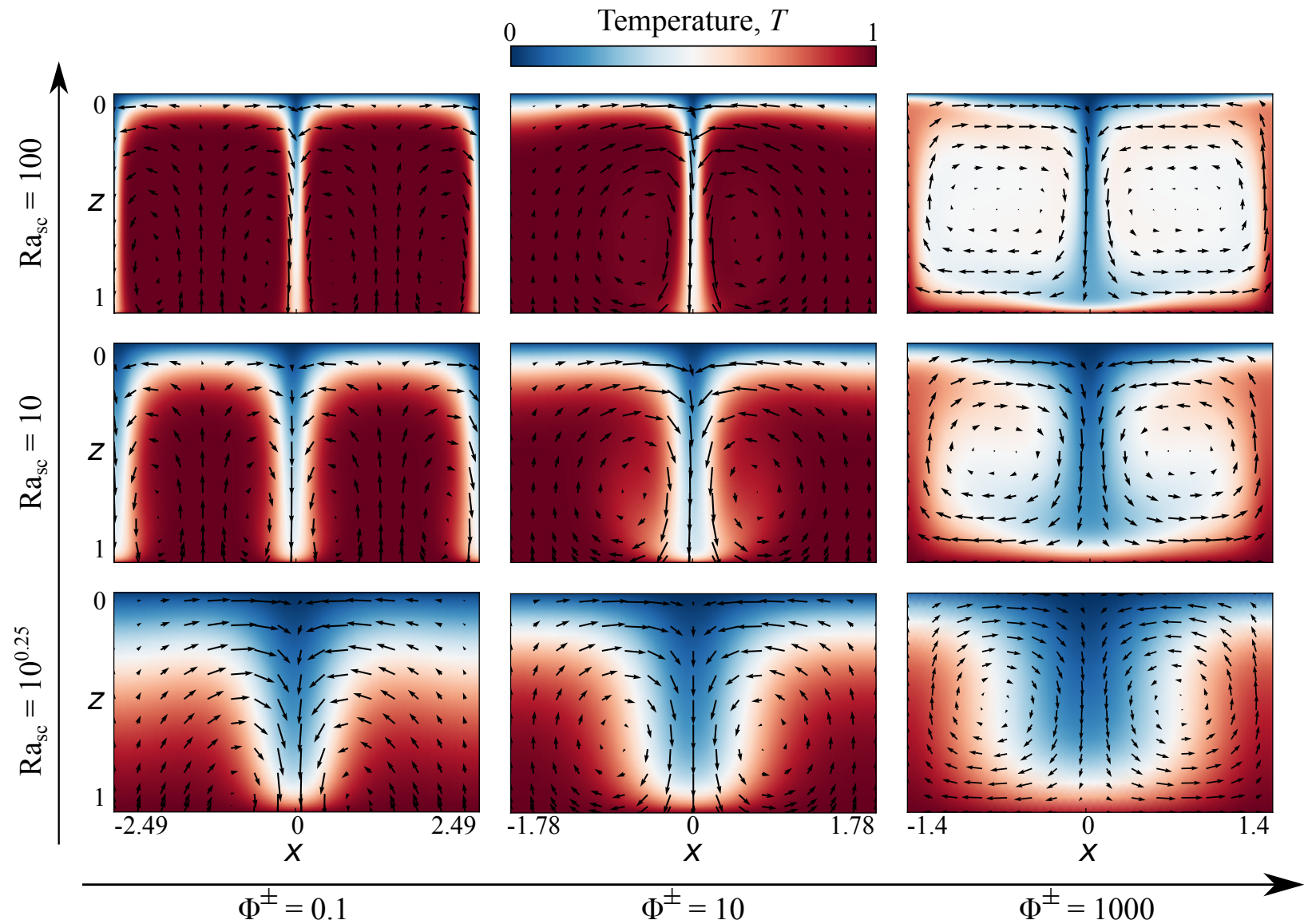

Figure 7. Convection patterns obtained with only a phase change only at the bottom boundary. Snapshots of temperature (colour) and velocity (arrows) for different case investigated. $\Phi^{-}$increases from left to right, and $R a_{s c}$ from the bottom to the top, as indicated by the axes. Note that the horizontal scale depends on the value of $\Phi^{-}$.
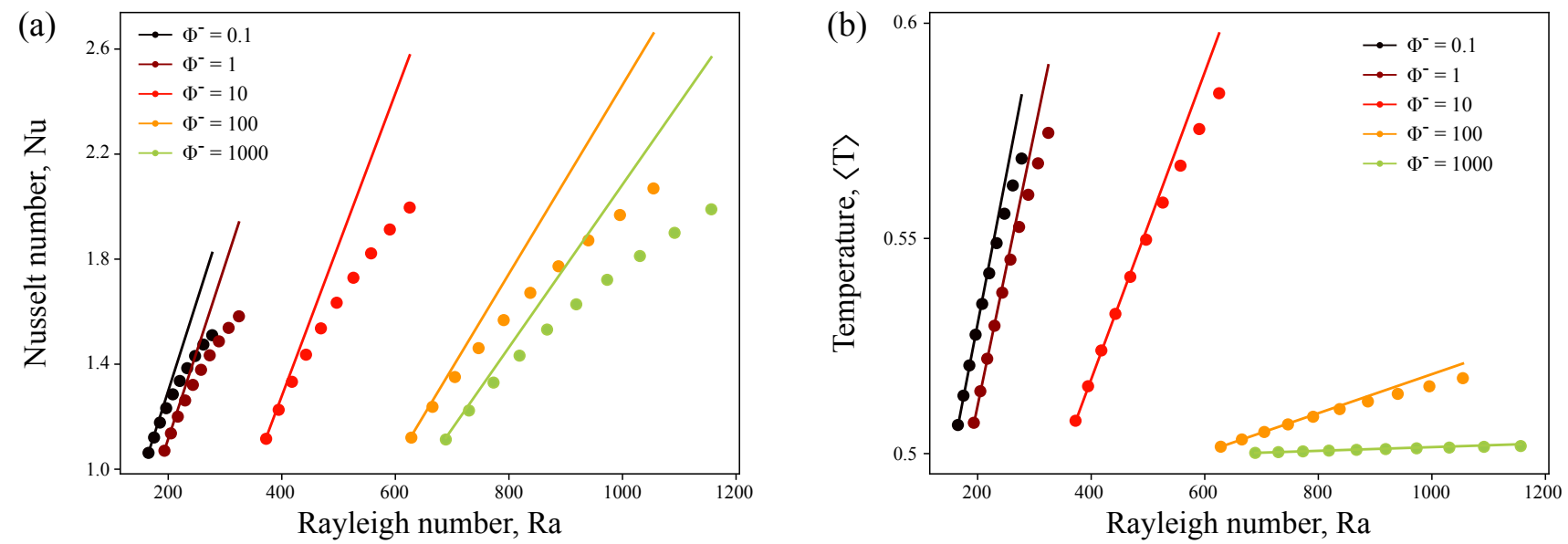

Figure 8. Heat flux and temperature close the critical value. (a) Nusselt number and (b) average temperature against the Rayleigh number. The lines represent the predictions of the weakly nonlinear analysis. 

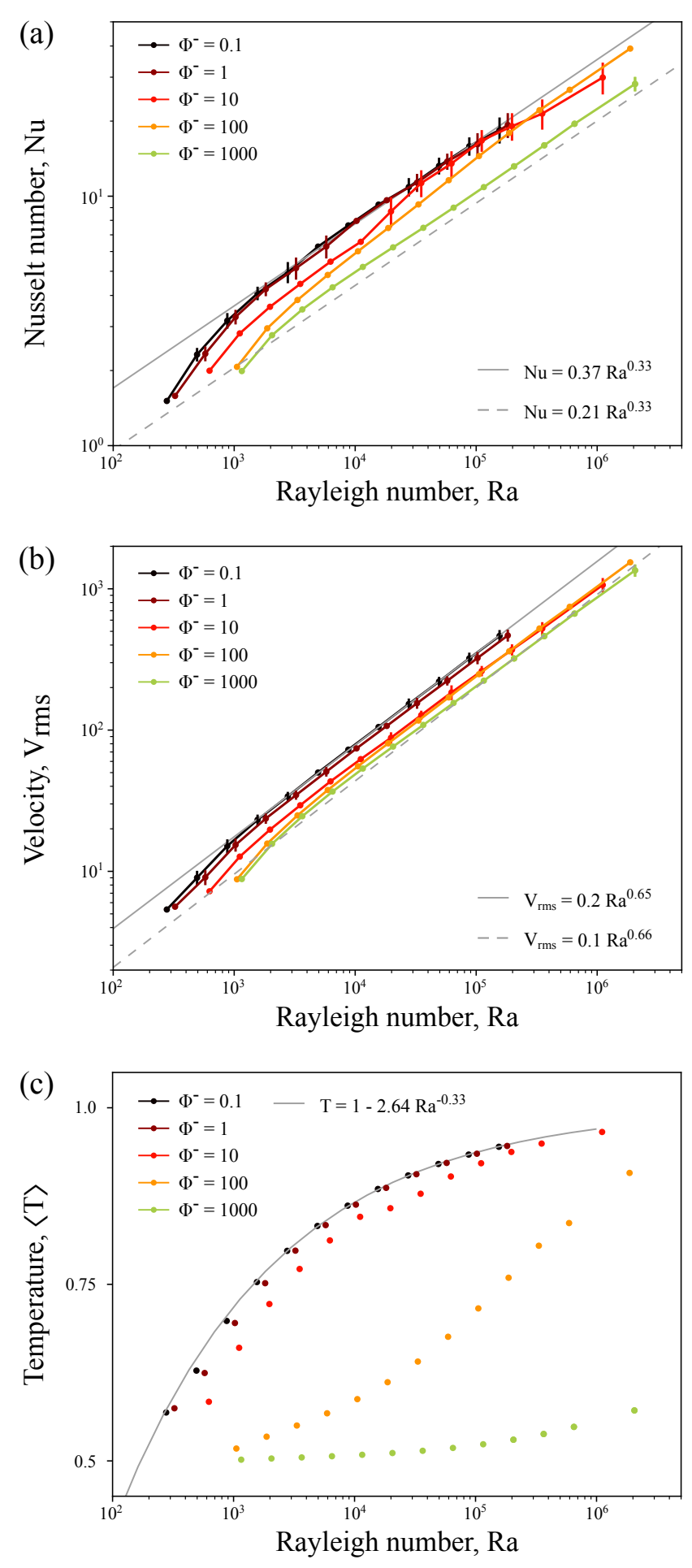

Figure 9. Heat and mass transfer characteristics for mantle convection with a solid-liquid phase change at the bottom. (a) Nusselt number, (b) RMS velocity, and (c) average temperature, against the Rayleigh number. The gray solid line represents the fit for the simulation for $\Phi^{-}=0.1$, and the grey dashed line the classical non-penetrative free-slip case as already shown on figure 5 . mantle may have occurred early in the history of Earth-or-largersized rocky Planets (Labrosse et al. 2007; Thomas et al. 2012; Boukaré et al. 2015). Assuming efficient mixing of the magma ocean, we expect the value of $\Phi$ to be less than 0.01 . In spherical geometry, the largest scale accessible to the flow is that of the spherical harmonics of degree 1 . The linear stability calculation of Morison et al. (2019) indeed predicts that convection should set in as a degree 1 mode of convection, i.e. a translation mode, for a mantle that has crystallised only a few hundreds of km. Applying the present results to that situation suggests that heat and mass transfer would rapidly grow to values that are orders of magnitude larger than any rate encountered in the solid mantle after full crystallisation of magma oceans. This would promote a heat flow from the deep interior to the surface magma ocean so large that the basal magma ocean and the core would cool faster than previously thought, possibly driving an early dynamo. Going beyond such simple conjectures requires however to take into account compositional effects that may significantly alter the dynamics of the solid mantle that is crystallising as it is convecting (e.g. Hess \& Parmentier 1995; Elkins-Tanton et al. 2003; Morison et al. 2019).

Considering now the case of only one magma ocean, the situation applies for the present time on icy satellites and possibly for a part of the history of the Earth (Labrosse et al. 2007). We only studied here the situation with a magma ocean below the solid mantle but the case with a magma ocean above is its symmetrical (fig. 6). The results presented above show that the form of convection and the thermal structure are dramatically modified and heat and mass transfer are greatly enhanced when phase melting and freezing occurs at one boundary, even though these effects are not as drastic as in the case of two phase change boundaries. In the case of a basal magma ocean that has been investigated thoroughly here, the dynamics and thermal structure bears many similarities with internally heated convection, with narrow cold plumes descending from the upper boundary and broad high temperature return flow elsewhere. Even though volumetric heating is not included in these calculation, the bottom boundary layer is completely suppressed and no hot plume can develop. This suggests that the dynamics of the surface ice shells of icy satellites and possibly of the early Earth mantle are entirely dominated by down-welling currents, leaving no role to hot plumes. In the case of the Earth mantle, the situation is certainly more complex with fractional crystallisation at the bottom possibly leading to compositional stratification, a situation that deserves further study.

In terms of heat transfer, we find that the dimensionless heat flux, the Nusselt number, scales with the Rayleigh number with an exponent equal to $1 / 3$, which is the same as for classical nonpenetrating conditions, but with a pre-factor about twice higher. This means that, for the same Rayleigh number, the thermal evolution with a basal magma ocean should be about twice faster than without, and this imply that thermal evolution models, involving a basal magma ocean, should take that effect into account. The parameterisation of the heat flow at the bottom of the solid mantle cannot rely on the existence of a boundary layer, as was assumed by Labrosse et al. (2007), since heat transfer happens by advection through the boundary. We expect however that, as compositionally dense material fractionally crystallizing at the bottom starts to accumulate (Labrosse et al. 2007), the dynamics of the bottom of the solid mantle strongly departs from the one shown here.

A magma ocean that simply cools by radiating heat into space would solidify completely in a few thousand years (Monteux et al. 2016). Considering the effect of a dense atmosphere can elongate this period to about $10 \mathrm{Myr}$ at Earth position (e.g. Abe 1997; 
Hamano et al. 2013; Lebrun et al. 2013; Salvador et al. 2017). Longer timescales can be reached for planets closer to their star (e.g. Hamano et al. 2013; Salvador et al. 2017). It is however difficult to explain with these models the apparent longevity of the Martian magma ocean (Debaille et al. 2007). Our result suggest that the possibility of phase change between the crystallising mantle and the magma ocean allows for a very efficient heat transfer by convection in the solid. This means that the contribution of the heat flow from the deepest part of the planet to the magma ocean thermal budget may not be as negligible as usually assumed. If the mantle crystallizes upward from the bottom and is in contact with only one magma ocean, the heat flux scaling obtained here would suggest a heat flow a factor of two larger than that obtained for classical non-penetrating conditions, for the same Rayleigh number. The importance of that heat flow depends then crucially on the values of poorly constrained parameters such as the viscosity of the solid mantle. On the other hand, it is quite possible that a basal magma ocean formed on Mars owing to the density inversion between olivine and silicate melt at about $8 \mathrm{GPa}$ (e.g. Ohtani 1983; Agee \& Walker 1993, 1988). In that case, the heat flow across the solid mantle could be orders of magnitude larger and contribute significantly to keep the magma ocean liquid. Heat is not the whole story in this scenario since fractional crystallisation would also lead to transfer of $\mathrm{FeO}$ between the top and basal magma oceans changing their freezing temperature. A full model including $\mathrm{FeO}$ exchange is therefore necessary to test whether this scenario could make the surface magma ocean live longer.

\section{ACKNOWLEDGEMENTS}

This research has been funded by the French Agence Nationale de la Recherche under the grant number ANR-15-CE31-0018-01, MaCoMaOc.

\section{REFERENCES}

Abe, Y., 1997. Thermal and chemical evolution of the terrestrial magma ocean, Phys. Earth Planet. Inter., 100, 27-39.

Agee, C. B. \& Walker, D., 1988. Mass balance and phase density constraints on early differentiation of chondritic mantle, Earth Planet. Sci. Lett., 90(2), 144-156.

Agee, C. B. \& Walker, D., 1993. Olivine flotation in mantle melt, Earth Planet. Sci. Lett., 114(2-3), 315-324.

Alboussière, T., Deguen, R., \& Melzani, M., 2010. Melting-induced stratification above the Earth's inner core due to convective translation, $\mathrm{Na}$ ture, 466, 744.

Amestoy, P., Duff, I., L'Excellent, J., \& Koster, J., 2001. A Fully Asynchronous Multifrontal Solver Using Distributed Dynamic Scheduling, SIAM J. Matrix Analysis and Applications, 23(1), 15-41.

Amestoy, P. R., Guermouche, A., L'Excellent, J.-Y., \& Pralet, S., 2006. Hybrid scheduling for the parallel solution of linear systems, Parallel Computing, 32(2), 136-156.

Baland, R.-M., Tobie, G., Lefèvre, A., \& Van Hoolst, T., 2014. Titan's internal structure inferred from its gravity field, shape, and rotation state, Icarus, 237, 29-41.

Ballmer, M. D., Lourenço, D. L., Hirose, K., Caracas, R., \& Nomura, R., 2017. Reconciling magma-ocean crystallization models with the presentday structure of the Earth's mantle, Geochem. Geophys. Geosyst., 18(7), 2785-2806.

Boukaré, C.-E., Ricard, Y., \& Fiquet, G., 2015. Thermodynamics of the $\mathrm{MgO}-\mathrm{FeO}-\mathrm{SiO} 2$ system up to $140 \mathrm{GPa}$ : Application to the crystallization of Earth's magma ocean, J. Geophys. Res., 120(9), 6085-6101.
Boukaré, C.-E., Parmentier, E., \& Parman, S., 2018. Timing of mantle overturn during magma ocean solidification, Earth Planet. Sci. Lett., 491, 216-225.

Čadek, O., Tobie, G., Van Hoolst, T., Massé, M., Choblet, G., Lefèvre, A., Mitri, G., Baland, R.-M., Běhounková, M., Bourgeois, O., \& Trinh, A., 2016. Enceladus's internal ocean and ice shell constrained from Cassini gravity, shape, and libration data, Geophys. Res. Letters, 43(11), 56535660 .

Chambat, F., Benzoni-Gavage, S., \& Ricard, Y., 2014. Jump conditions and dynamic surface tension at permeable interfaces such as the inner core boundary, C. R. Geosciences, 346(5-6), 110-118.

Debaille, V., Brandon, A. D., Yin, Q. Z., \& Jacobsen, B., 2007. Coupled ${ }^{142} \mathrm{Nd}-{ }^{143} \mathrm{Nd}$ evidence for a protracted magma ocean in mars, Nature, 450, 525 .

Deguen, R., 2013. Thermal convection in a spherical shell with melting/freezing at either or both of its boundaries, J. Earth Sci., 24, 669682.

Deguen, R., Alboussière, T., \& Cardin, P., 2013. Thermal convection in Earth's inner core with phase change at its boundary, Geophys. J. Int., 194(3), 1310-1334.

Deguen, R., Alboussière, T., \& Labrosse, S., 2018. Double-diffusive translation of Earth's inner core, Geophys. J. Int..

Elkins-Tanton, L. T., 2012. Magma Oceans in the Inner Solar System, Ann. Rev. Earth Planet. Sci., 40(1), 113-139.

Elkins-Tanton, L. T., Parmentier, E. M., \& Hess, P. C., 2003. Magma ocean fractional crystallization and cumulate overturn in terrestrial planets: Implications for Mars, Meteoritics \& Planetary Science, 38(12), 1753-1771.

Grasset, O., Sotin, C., \& Deschamps, F., 2000. On the internal structure and dynamics of Titan, Planet. Spa. Sci., 48(7), 617-636.

Grigné, C., Labrosse, S., \& Tackley, P. J., 2005. Convective heat transfer as a function of wavelength: Implications for the cooling of the Earth, $J$. Geophys. Res., 110(B3), B03409.

Hamano, K., Abe, Y., \& Genda, H., 2013. Emergence of two types of terrestrial planet on solidification of magma ocean, Nature, 497, 607.

Hess, P. \& Parmentier, E., 1995. A model for the thermal and chemical evolution of the Moon's interior: implications for the onset of mare volcanism, Earth Planet. Sci. Lett., 134(3), 501 - 514.

Houseman, G., 1988. The dependence of convection planform on mode of heating, Nature, 332(6162), 346-349.

Jaupart, C. \& Mareschal, J.-C., 2011. Heat generation and transport in the Earth, Cambridge University Press, Cambridge.

Khurana, K. K., Kivelson, M. G., Stevenson, D. J., Schubert, G., Russell, C. T., Walker, R. J., \& Polanskey, C., 1998. Induced magnetic fields as evidence for subsurface oceans in Europa and Callisto, Nature, 395, 777.

Labrosse, S., Hernlund, J. W., \& Coltice, N., 2007. A crystallizing dense magma ocean at the base of the Earth's mantle, Nature, 450, 866.

Labrosse, S., Morison, A., Deguen, R., \& Alboussière, T., 2018. Rayleigh-Bénard convection in a creeping solid with melting and freezing at either or both its horizontal boundaries, J. Fluid Mech., 846, 5-36.

Lebrun, T., Massol, H., Chassefière, E., Davaille, A., Marcq, E., Sarda, P., Leblanc, F., \& Brandeis, G., 2013. Thermal evolution of an early magma ocean in interaction with the atmosphere, J. Geophys. Res., 118(6), 1155-1176.

Maurice, M., Tosi, N., Samuel, H., Plesa, A.-C., Hüttig, C., \& Breuer, D., 2017. Onset of solid-state mantle convection and mixing during magma ocean solidification, J. Geophys. Res., 122(3), 577-598.

Mizzon, H. \& Monnereau, M., 2013. Implication of the lopsided growth for the viscosity of Earth's inner core, Earth Planet. Sci. Lett., 361, 391 -401 .

Monnereau, M., Calvet, M., Margerin, L., \& Souriau, A., 2010. Lopsided Growth of Earth's Inner Core, Science, 328(5981), 1014 LP - 1017.

Monteux, J., Andrault, D., \& Samuel, H., 2016. On the cooling of a deep terrestrial magma ocean, Earth Planet. Sci. Lett., 448, 140-149.

Morison, A., Labrosse, S., Deguen, R., \& Alboussière, T., 2019. Timescale of overturn in a magma ocean cumulate, Earth Planet. Sci. Lett., 516, 25-36.

Ohtani, E., 1983. Melting temperature distribution and fractionation in the 
lower mantle, Phys. Earth Planet. Inter., 33(1), 12-25.

Pappalardo, R. T., Head, J. W., Greeley, R., Sullivan, R. J., Pilcher, C., Schubert, G., Moore, W. B., Carr, M. H., Moore, J. M., Belton, M. J. S., \& Goldsby, D. L., 1998. Geological evidence for solid-state convection in Europa\&\#39;s ice shell, Nature, 391, 365.

Parmentier, E. M., Sotin, C., \& Travis, B. J., 1994. Turbulent 3-D thermal convection in an infinite Prandtl number, volumetrically heated fluid: implications for mantle dynamics, Geophysical Journal International, 116(2), 241-251.

Ricard, Y., Labrosse, S., \& Dubuffet, F., 2014. Lifting the cover of the cauldron: Convection in hot planets, Geochem. Geophys. Geosyst., 15(12), 4617-4630.

Salvador, A., Massol, H., Davaille, A., Marcq, E., Sarda, P., \& Chassefière, E., 2017. The relative influence of $\mathrm{H}_{2} \mathrm{O}$ and $\mathrm{CO}_{2}$ on the primitive surface conditions and evolution of rocky planets, J. Geophys. Res., 122(7), $1458-1486$.

Schubert, G., Turcotte, D. L., \& Olson, P., 2001. Mantle Convection in the Earth and Planets, Cambridge University Press, Cambridge.

Solomatov, V., 2015. 9.04 - Magma Oceans and Primordial Mantle Differentiation, pp. 81-104, ed. Schubert, G. B. T. T. o. G. S. E., Elsevier, Oxford.

Sotin, C. \& Labrosse, S., 1999. Three-dimensional thermal convection in an iso-viscous, infinite Prandtl number fluid heated from within and from below: applications to the transfer of heat through planetary mantles, Phys. Earth Planet. Inter, 112(3-4), 171-190.

Tackley, P. J., 1998. Self-consistent generation of tectonic plates in threedimensional mantle convection, Earth Planet. Sci. Lett., 157(1), 9-22.

Thomas, C. W., Liu, Q., Agee, C. B., Asimow, P. D., \& Lange, R. A., 2012. Multi-technique equation of state for $\mathrm{Fe} 2 \mathrm{SiO} 4 m e l t$ and the density of $\mathrm{Fe}-$ bearing silicate melts from 0 to $161 \mathrm{GPa}, J$. Geophys. Res., 117(B10).

Tobie, G., Choblet, G., \& Sotin, C., 2003. Tidally heated convection: Constraints on Europa's ice shell thickness, J. Geophys. Res., 108(E11). 